\title{
Production of stunted pot plants from Ruellia simplex
}

\section{T.M. Noor El-Deen}

Ornamental Plants and Landscape Gardening Res. Dept., Hort. Res. Inst., ARC, Giza, Egypt. Received: 30 Jan. 2020/Accepted 20 April 2020 / Publication date: 30 April 2020

\begin{abstract}
A pot experiment was carried out at Botanical Garden of National Gene Bank, Agricultural Research Center, Giza, Egypt during 2018 and 2019 seasons to investigate the effect of pinching treatments and foliar spraying with paclobutrazol (PBZ) on Mexican petunia (Ruellia simplex C.Wright), aiming to produce more compact plants. Three pinching treatments (non, single after 1 month from transplanting and double after 1 and 3 months from transplanting) and 4 PBZ concentrations of $0,50,100$ and $150 \mathrm{ppm}$ at one-month interval and their combined treatments laid out as a factorial RCBD was employed in this study. Best single-treatment results of dense foliage were found with single pinching or PBZ at either 50 or $100 \mathrm{ppm}$. As for combined effects, single pinching + spraying with PBZ at $100 \mathrm{ppm}$ was the superior combined treatment; with a reduced plant height by 50.17 and $50.06 \%$, increased number of branches/plant by 95.85 and $96.18 \%$, reduced number of leaves/plant by only 10.27 and $9.26 \%$ and increased number of flowers/plant by 51.97 and $48.43 \%$ in both seasons, respectively. However, this treatment delayed flowering by 5.3 days in the first season only. The highest values regarding chlorophylls (a and b), carotenoids and total indoles were recorded by this superior combined treatment. Finally, it is recommended to treat Mexican petunia plants cultivated in 16-cm-pots filled with peat moss + perlite (1:1 by volume) with single pinch after 1 month from transplanting +4 applications at one-month interval with PBZ at $100 \mathrm{ppm}$ for optimum stunting with satisfied quality as pot plant.
\end{abstract}

Keywords: Ruellia simplex, stunting, pinching, paclobutrazol, vegetative growth, flowering.

\section{Introduction}

Ruellia (Acanthaceae) is a flowering plant genus comprised of about 250 perennial herb, subshrub, and shrub species with mostly tropical and subtropical distribution. Ruellia simplex C.Wright (synonyms: $R$. tweediana, $R$. brittoniana, $R$. malacosperma, $R$. coerulea) is commonly known as Mexican petunia or Mexican bluebell and is native to Mexico, southern North America, southern South America, and the Antilles. It is characterized by linear to lanceolate leaves with very prominent veins, oppositely arranged along the stem. Green to purple stems with more than $90 \mathrm{~cm}$ height have prominent nodal swellings. The purple, pedunculate flowers are funnel-shaped and have five petals that are either solitary or appear in a few-flowered cymes arising where the leaves attach to the stem. Plants can bloom throughout the year while, peak flowering has been observed in spring and summer months (Wiese et al., 2013; Freyre and Wilson, 2014 and Smith, et al., 2010).

Manipulating plant growth for an aesthetically marketable form is an integral part of the program of many flower crops. Besides plant growth regulators as chemical retardants, there are a number of nonchemical (biological and physical) control options to manipulate plant growth so that well-proportioned compact plants are produced. In this regard, container size, timing, water stress, nutrient stress, light, temperature and pinching are considered as physical control (Nau, 2011).

Pinching is one of the most common and efficacious tactics for successful cultivation of cut flowers as well as potted plants (Cline, 1991). Pinching is the removal of the terminal growing portion, this led to reduce height but promotes auxiliary branches, delays flowering and helps in breaking resting period (Sehrawat et al., 2003). When the apical portion of the shoot is removed, large number of auxiliary shoots arise resulting in well-shaped bushy plants bearing great number of uniform flowers as tested on African marigold (Khandelval et al., 2003). Pinching plants is used to promote lateral branching, but a secondary effect is the reduction of plant height (Heins et al., 2000). A very common industry practice, research has shown confirmation of the desired effect of pinching, as reported by many authors e.g. Gibson et al. (2003) on Argyranthemum frutescens, Saiyad et al. (2010) on Gaillardia, Shanan and Soliman (2011) on snapdragon, Rezazadeh and Harkess (2015) on

Corresponding Author: T.M. Noor El-Deen, Ornamental Plants and Landscape Gardening Res. Dept., Hort. Res. Inst., ARC, Giza, Egypt. E-mail: tarek.noor@arc.sci.eg 
Odontonema callistachyum, Vasoya et al. (2015) on Gaillardia, Rathore and Mishra (2014) on Tagetes erecta L., Abou-Dahab et al. (2015) on Russelia equisetiformis and El-Sadek (2016) on Hibiscus rosa-sinensis, L. cv. "Yellow".

Paclobutrazol (PBZ) is a chemical used widely in agriculture and horticulture as a plant growth regulator. In ornamental crops, PBZ is used to reduce the size of plants, improve compactness and increase other functional aspects, such as resistance to both abiotic and biotic stresses (Bañón et al., 2009). Foliar spraying with PBZ is applied mainly to control plant height of many floriculture crops, for example, it could be sprayed at 5 to $45 \mathrm{ppm}$ for ageratum, 100 to $200 \mathrm{ppm}$ for azalea and caladium, 6 to $66 \mathrm{ppm}$ for bedding plants, 4 to $50 \mathrm{ppm}$ for celosia, 50 to 200 for potted chrysanthemum, 5 to 45 ppm for coleus and dahlia, 5 to 60 for dianthus, 100 to 300 for freesia and 10 to 30 for poinsettia (Bailey, 1998). Positive effects of PBZ were reported by many authors as Maus (1987) on Hibiscus rosa-sinensis, Villegas and Lozoya (1992) on poinsettia, Wilfret and Barrett (1995) on Rhododendron obtusum, Gad et al. (1997) on Fuchsia magellanica cv. Beacon, El-Sallami (2001) on potted poinsettia, Warner and Erwin (2003) on Hibiscus coccineus, H. radiatus and $H$. trionum, Matsoukis et al. (2004) on Lantana camara, Meijón et al. (2009) on Azalea japonica and Saiyad et al. (2010) and Vasoya et al. (2015) on gaillardia, Shanan and Soliman (2011) on snapdragon, Rathore and Mishra (2014) on Tagetes erecta L., Shahin et al. (2014) on Chrysanthemum carinatum, Mutlu and Agan (2015) on ornamental pepper, El-Sadek (2016) on Hibiscus rosa-sinensis and Mohamed (2016) on Lagerstroemia indica.

Few studies have been carried out concerning the ability of producing dwarf Mexican petunia as a commercial potted plant, one of these studies utilized daminozide at different concentrations $(0$ $4000 \mathrm{ppm}$ ) as a growth retardant, the obtained results revealed that both plant height, leaf area, number of flowers/plant, number of leaves/plant were decreased with increasing daminozide concentration (Nurhazwani et al., 2018).

The present study was conducted to investigate the effect of pinching treatments (non, single and double) and foliar spraying with PBZ at different concentrations (0, 50, 100 and $150 \mathrm{ppm})$ on $R$. simplex plants to produce stunted commercial pot plants with high quality and aesthetic value.

\section{Materials and Methods}

A pot experiment was carried out at the Botanical Garden of the National Gene Bank, Agricultural Research Center, Giza, Egypt during 2018 and 2019 seasons to investigate the effect of pinching treatments and foliar spraying with PBZ on Ruellia simplex plants to produce stunted pot plants without observed reduction in quality.

\section{Plant material:}

About 15-cm-terminal cuttings of $R$. simplex were collected from National Gene Bank Botanical Garden on March, $25^{\text {th }}$ and March, $24^{\text {th }}$ for the first and second seasons, respectively, and cultivated in 10-cm-pots filled with peat moss + perlite $(1: 1$, by volume). Cultivated cuttings were kept in the National Gene Bank greenhouse at $23 \pm 2{ }^{\circ} \mathrm{C}$ air temperature, $60-65 \%$ relative humidity and 4000 lux light intensity to ensure good establishment. After 35 days (April, 29 ${ }^{\text {th }}$ and April, 30 $0^{\text {th }}$ for the first and second seasons, respectively), successfully rooted cuttings were transplanted into $16-\mathrm{cm}$-pots filled with about $225 \mathrm{~g}$ of peat moss + perlite (1:1, by volume). After a fifteen days re-establishment period, plants were transferred to the experimental field site to be kept until the beginning of experiment treatments. Meteorological parameters at Giza Governorate during 2018 and 2019 seasons are shown in Table (1).

\section{Experimental layout:}

This experiment was laid out as a randomized complete block design (RCBD) in a factorial experiment (Gomez and Gomez, 1984) with two factors; three pinching treatments (non, single and double pinching) represented factor $\mathrm{A}$, while 4 foliar spraying with paclobutrazol $(0,50,100$ and 150 $\mathrm{ppm}$ ) represented factor B. So, this experiment contained 12 treatments, each treatment was replicated three times, each replicate contained 6 pots. 


\section{Pinching treatments:}

After one month from transplanting (two weeks from transferring to the experiment site) on May, $27^{\text {th }}$ and $29^{\text {th }}$ for the first and second seasons, respectively, plants were divided into three equal groups: the first one has been left without pinching (non-pinching), the second one was pinched one time only by removing about $2 \mathrm{~cm}$ of shoot tips (single pinching), while the third one was pinched two times; the first one was done at the same date of single pinching, while the second pinching was done after three months from transplanting (on July, $29^{\text {th }}$ and $28^{\text {th }}$ for the first and second seasons, respectively) by removing about $2 \mathrm{~cm}$ of shoot tips of the new growth obtained from the first pinching (double pinching).

Table 1: Some meteorological parameters at Giza Governorate during 2018 and 2019 seasons.

\begin{tabular}{|c|c|c|c|c|c|c|c|c|c|c|}
\hline \multirow{3}{*}{ Months } & \multicolumn{5}{|c|}{2018} & \multicolumn{5}{|c|}{2019} \\
\hline & \multicolumn{3}{|c|}{ Temperature $\left({ }^{\circ} \mathrm{C}\right)$} & \multirow{2}{*}{$\begin{array}{l}\text { R.H. } \\
(\%)\end{array}$} & \multirow{2}{*}{$\begin{array}{c}\text { W.S. } \\
(\mathbf{m} / \mathbf{s e c})\end{array}$} & \multicolumn{3}{|c|}{ Temperature $\left({ }^{\circ} \mathrm{C}\right)$} & \multirow{2}{*}{$\begin{array}{l}\text { R.H. } \\
(\%)\end{array}$} & \multirow{2}{*}{$\begin{array}{c}\text { W.S. } \\
\text { (m/sec) }\end{array}$} \\
\hline & Max & Min & Average & & & Max & Min & Average & & \\
\hline April & 29.87 & 15.93 & 25.03 & 42.67 & 1.63 & 28.53 & 14.47 & 23.87 & 46.33 & 2.07 \\
\hline May & 35.07 & 21.07 & 30.03 & 41.67 & 1.90 & 35.53 & 19.03 & 30.23 & 25.83 & 2.30 \\
\hline June & 36.45 & 28.05 & 31.45 & 41.50 & 2.10 & 38.00 & 23.17 & 32.10 & 41.00 & 2.20 \\
\hline July & 37.93 & 24.43 & 32.63 & 45.67 & 1.93 & 38.17 & 24.27 & 33.13 & 42.67 & 2.17 \\
\hline August & 37.13 & 25.13 & 32.10 & 47.00 & 1.97 & 38.99 & 22.73 & 30.53 & 39.77 & 2.64 \\
\hline September & 35.60 & 24.10 & 31.07 & 48.00 & 1.97 & 35.97 & 20.69 & 27.64 & 47.56 & 2.83 \\
\hline
\end{tabular}

* These parameters were collected and averaged from the data obtained from Soil, Water \& Environment Research Institute, ARC and NASA Power Data Access Viewer Program (https://power.larc.nasa.gov).

* No precipitation was observed during this period except for April in 2019 only which recoded $1.8 \mathrm{~mm}$.

\section{Foliar spraying with paclobutrazol (PBZ):}

PBZ $\left(\mathrm{C}_{15} \mathrm{H}_{20} \mathrm{ClN}_{3} \mathrm{O}\right.$, MW: 293.80) manufactured in USA was obtained from a local company in Egypt. Solutions were prepared by dissolving paclobutrazol in distilled water to obtain the required concentrations i.e. 50, 100 and $150 \mathrm{ppm}$. In this regard, PBZ at $0 \mathrm{ppm}$ (spraying with distilled water only) refers to control. Four applications of each paclobutrazol concentration at one-month interval were applied per season during the course of this study. The first spray application was done after about 2 weeks from the first pinching (on June, $12^{\text {th }}$ and $13^{\text {th }}$ for the first and second seasons, respectively). Plants were foliar sprayed with each concentration till run off.

Likewise, the interaction treatments between 3 pinching treatments and 4 paclobutrazol concentrations were done, so the total number of treatments applied in this study were 12 treatments as described above.

\section{Data recorded:}

At the end of September for each season, the following data were recorded:

\section{Vegetative growth and root parameters:}

Plant height $(\mathrm{cm})$, number of branches/plant, vegetative parts fresh weight $(\mathrm{g})$, vegetative parts dry weight $(\mathrm{g})$, number of leaves/plant and leaf area $\left(\mathrm{cm}^{2}\right)$. In this regard, fresh leaf samples were collected and scanned at a resolution of 300 dpi by using digital scanner then the leaf area was calculated by using ImageJ software as described by Ferreira and Rasband (2012). Moreover, root length $(\mathrm{cm})$, roots fresh and dry weights $(\mathrm{g})$ were measured.

\section{Flowering parameters:}

Number of days to flowering (days) was counted from transplanting date to the first flower opening of each plant, number of flowers/plant during the course of this study (till the end of September) and flower corolla diameter (width; $\mathrm{mm}$ ) were measured carefully to avoid distorting the flower by placing the ruler over the face of the flower and measure its greatest width/diameter across two tips of the lobes. 


\section{Chemical constituents:}

At the end of September in the second season the following chemical tests were performed: pigments content (mg/g f.w.) as chlorophylls ( $\mathrm{a}$ and $\mathrm{b}$ ) and carotenoids were determined in fresh leaf samples according to Wellburn and Lichtenthaler (1984), total carbohydrates percentage was determined in either dry shoots and root samples according to the method described by Herbert et al. (1971), total indoles in dry shoots $(\mathrm{mg} / 100 \mathrm{~g}$ d.w.) was determined according to the method described by Larsen et al. (1962) and total phenols in dry shoots (\%) according to A.O.A.C. (1980).

\section{Statistical analysis:}

The obtained data were statistically analyzed using MSTAT Computer Program (MSTAT Development Team, 1989). To verify differences among means of various treatments, means were compared using Duncan's Multiple Range Test as described by Duncan (1955).

\section{Results and Discussion}

\section{Effect of pinching and PBZ treatments on:}

\section{Vegetative growth and root parameters:}

Significant differences were observed due to applying different pinching and PBZ treatments on vegetative and root growth parameters of $R$. simplex plants as shown in Tables (2 and 3).

Regarding the effect of pinching treatments, non-pinching produced the tallest plants (45.63 and $48.77 \mathrm{~cm})$, the heaviest vegetative parts fresh weight $(20.17$ and $22.00 \mathrm{~g})$, the biggest leaf area $(5.11$ and $\left.5.32 \mathrm{~cm}^{2}\right)$ and the longest roots $(76.82$ and $81.86 \mathrm{~cm})$ in both seasons, respectively. However, the highest number of branches/plant was obtained by double pinching as recorded 5.30 and 5.22 in both seasons, respectively. In this regard, the highest vegetative parts dry weight in the second season (6.03 $\mathrm{g}$ ), the highest number of leaves/plant in both seasons (30.48 and 32.30, respectively), the highest roots fresh weight in both seasons (11.88 and $14.37 \mathrm{~g}$, respectively) and the highest roots dry weight in both seasons (3.62 and $4.64 \mathrm{~g}$, respectively) were obtained by single pinching. On the other hand, the highest reduction in plant height was obtained by double pinching as recorded 34.48 and $35.08 \mathrm{~cm}$ in both seasons, respectively. In addition, this treatment caused a maximum reduction in some studied traits i.e. vegetative parts fresh and dry weights, leaf area and root length.

As for the effect of PBZ concentrations, plants sprayed with PBZ at $0 \mathrm{ppm}$ (control) significantly produced the highest values in most cases i.e. plant height $(55.78$ and $57.78 \mathrm{~cm})$, vegetative parts fresh weight (24.13 and $25.36 \mathrm{~g})$, vegetative parts dry weight $(6.01$ and $6.90 \mathrm{~g})$, number of leaves/plant (34.43 and 36.24), leaf area (5.86 and $6.33 \mathrm{~cm} 2)$, root length (77.61 and 81.47 $\mathrm{cm})$, roots fresh weight (13.61 and $16.32 \mathrm{~g}$ ) and roots dry weight (3.63 and $5.10 \mathrm{~g})$ in both seasons, respectively.

On the other hand, the highest reduction was obtained by spraying with the highest level of PBZ $(150 \mathrm{ppm})$ in case of plant height $(20.40$ and $21.67 \mathrm{~cm})$, vegetative parts fresh weight $(12.12$ and $14.19 \mathrm{~g})$, vegetative parts dry weight (3.22 and $3.86 \mathrm{~g}$ ), number of leaves/plant (21.69 and 22.66), leaf area $\left(3.71\right.$ and $\left.4.06 \mathrm{~cm}^{2}\right)$, root length $(63.60$ and $68.93 \mathrm{~cm})$, roots fresh weight $(8.18$ and $10.68 \mathrm{~g})$ and roots dry weight $(2.42$ and $3.05 \mathrm{~g})$ in both seasons, respectively. Regarding the number of branches/plant the highest values (6.29 and 6.67 in both seasons, respectively) were obtained by PBZ at $100 \mathrm{ppm}$, while the maximum reduction (3.51 and 4.09 in both seasons, respectively) was obtained without PBZ spraying.

Respecting the effect of the interaction treatments between pinching and PBZ concentrations, it is clear that single pinching $+\mathrm{PBZ}$ at $0 \mathrm{ppm}$ (spraying with distilled water only) significantly produced the highest values in case of vegetative parts fresh weight (24.61 and $26.08 \mathrm{~g}$ ), vegetative parts dry weight (6.32 and 7.51 g), number of leaves/plant (37.11 and 39.23), leaf area (6.08 and 6.50 $\mathrm{cm}^{2}$ ), roots fresh weight (14.30 and $\left.17.62 \mathrm{~g}\right)$ and roots dry weight (4.29 and $\left.5.83 \mathrm{~g}\right)$ in both seasons, respectively. The mastery was to the combined treatment of non-pinching $+\mathrm{PBZ}$ at $0 \mathrm{ppm}$ (control) in terms of plant height $(61.33$ and $62.67 \mathrm{~cm})$ and root length $(84.00$ and $87.44 \mathrm{~cm})$ in both seasons respectively. In this regard, the highest number of branches/plant was obtained by double pinching + spraying with PBZ at $100 \mathrm{ppm}$ (7.07 and 7.40 in both seasons, respectively). On the contrary, the maximum reduction of most studied traits was obtained by double pinching + spraying with PBZ at 
$150 \mathrm{ppm}$ except for the number of branches/plant. Furthermore, it could be also observed that single pinching + spraying with PBZ at 100 ppm mediated other combined treatments and produced moderate values of these studied traits with optimum reduction.

Table 2: Effect of pinching treatments, PBZ concentrations and their interactions on some vegetative growth parameters of Ruellia simplex plants during 2018 and 2019 seasons.

\begin{tabular}{|c|c|c|c|c|c|c|c|c|}
\hline \multirow{3}{*}{$\begin{array}{c}\text { PBZ } \\
\text { concentrations } \\
\text { (B) }\end{array}$} & \multicolumn{4}{|c|}{ Pinching (A) } & \multicolumn{4}{|c|}{ Pinching (A) } \\
\hline & Non & Single & Double & Mean (B) & Non & Single & Double & Mean (B) \\
\hline & \multicolumn{4}{|c|}{2018} & \multicolumn{4}{|c|}{2019} \\
\hline & \multicolumn{8}{|c|}{ Plant height $(\mathrm{cm})$} \\
\hline 0 ppm & $61.33 \mathrm{a}$ & $53.47 \mathrm{~b}$ & $52.53 \mathrm{~b}$ & $55.78 \mathrm{a}$ & $62.67 \mathrm{a}$ & $56.40 \mathrm{~b}$ & $54.27 \mathrm{c}$ & $57.78 \mathrm{a}$ \\
\hline 50 ppm & $52.40 \mathrm{~b}$ & $47.20 \mathrm{c}$ & $42.13 \mathrm{~d}$ & $47.24 \mathrm{~b}$ & $57.33 \mathrm{~b}$ & $48.40 \mathrm{~d}$ & $44.33 \mathrm{e}$ & $50.02 \mathrm{~b}$ \\
\hline 100 ppm & $42.20 \mathrm{~d}$ & $30.56 \mathrm{e}$ & $27.00 \mathrm{f}$ & $33.25 \mathrm{c}$ & $47.27 \mathrm{~d}$ & $31.30 \mathrm{f}$ & $23.48 \mathrm{~h}$ & $34.02 \mathrm{c}$ \\
\hline 150 ppm & $26.60 \mathrm{f}$ & $18.33 \mathrm{~g}$ & $16.26 \mathrm{~h}$ & $20.40 \mathrm{~d}$ & $27.80 \mathrm{~g}$ & $19.00 \mathrm{i}$ & $18.22 \mathrm{i}$ & $21.67 \mathrm{~d}$ \\
\hline \multirow[t]{2}{*}{ Mean (A) } & $45.63 \mathrm{a}$ & $37.39 \mathrm{~b}$ & $34.48 \mathrm{c}$ & & $48.77 \mathrm{a}$ & $38.78 \mathrm{~b}$ & $35.08 \mathrm{c}$ & \\
\hline & \multicolumn{8}{|c|}{ Number of branches/plant } \\
\hline 0 ppm & $3.13 \mathrm{~g}$ & $3.53 \mathrm{fg}$ & 3.87 ef & $3.51 \mathrm{~d}$ & $3.40 \mathrm{~g}$ & 4.00 ef & $4.87 \mathrm{~d}$ & $4.09 \mathrm{~d}$ \\
\hline 50 ppm & $3.40 \mathrm{fg}$ & $3.67 \mathrm{fg}$ & $4.93 \mathrm{~d}$ & $4.00 \mathrm{c}$ & $3.87 \mathrm{fg}$ & $4.33 \mathrm{~d}-\mathrm{f}$ & $5.80 \mathrm{c}$ & $4.67 \mathrm{c}$ \\
\hline 100 ppm & $5.67 \mathrm{bc}$ & $6.13 \mathrm{~b}$ & $7.07 \mathrm{a}$ & $6.29 \mathrm{a}$ & $5.93 \mathrm{c}$ & $6.67 \mathrm{~b}$ & $7.40 \mathrm{a}$ & $6.67 \mathrm{a}$ \\
\hline 150 ppm & 3.80 ef & $4.33 \mathrm{e}$ & $5.33 \mathrm{~cd}$ & $4.49 \mathrm{~b}$ & $4.53 \mathrm{de}$ & $5.87 \mathrm{c}$ & $6.33 \mathrm{bc}$ & $5.58 \mathrm{~b}$ \\
\hline \multirow[t]{2}{*}{ Mean (A) } & $4.00 \mathrm{c}$ & $4.42 \mathrm{~b}$ & $5.30 \mathrm{a}$ & & $4.43 \mathrm{c}$ & $5.22 \mathrm{~b}$ & $6.10 \mathrm{a}$ & \\
\hline & \multicolumn{8}{|c|}{ Vegetative parts fresh weight (g) } \\
\hline 0 ppm & $24.17 \mathrm{a}$ & $24.61 \mathrm{a}$ & $23.61 \mathrm{a}$ & $24.13 \mathrm{a}$ & $25.12 \mathrm{bc}$ & $26.08 \mathrm{a}$ & $24.89 \mathrm{c}$ & $25.36 \mathrm{a}$ \\
\hline 50 ppm & $21.18 \mathrm{bc}$ & $22.22 \mathrm{~b}$ & $20.21 \mathrm{~cd}$ & $21.20 \mathrm{~b}$ & $23.53 \mathrm{~d}$ & $25.81 \mathrm{ab}$ & $23.91 \mathrm{~d}$ & $24.42 \mathrm{~b}$ \\
\hline 100 ppm & $20.25 \mathrm{~cd}$ & $19.35 \mathrm{de}$ & $18.71 \mathrm{e}$ & $19.44 \mathrm{c}$ & $22.50 \mathrm{e}$ & $21.57 \mathrm{f}$ & $21.33 \mathrm{f}$ & $21.80 \mathrm{c}$ \\
\hline 150 ppm & $15.09 \mathrm{f}$ & $11.24 \mathrm{~g}$ & $10.03 \mathrm{~h}$ & $12.12 \mathrm{~d}$ & $16.85 \mathrm{~g}$ & $13.43 \mathrm{~h}$ & $12.29 \mathrm{i}$ & $14.19 \mathrm{~d}$ \\
\hline \multirow[t]{2}{*}{ Mean (A) } & $20.17 \mathrm{a}$ & $19.35 \mathrm{~b}$ & $18.14 \mathrm{c}$ & & $22.00 \mathrm{a}$ & $21.72 \mathrm{a}$ & $20.60 \mathrm{~b}$ & \\
\hline & \multicolumn{8}{|c|}{ Vegetative parts dry weight (g) } \\
\hline $\mathbf{0} \mathbf{~ p p m}$ & $6.21 \mathrm{a}$ & $6.32 \mathrm{a}$ & $5.50 \mathrm{~b}$ & $6.01 \mathrm{a}$ & $7.04 \mathrm{~b}$ & $7.51 \mathrm{a}$ & $6.15 \mathrm{c}$ & $6.90 \mathrm{a}$ \\
\hline 50 ppm & $4.99 \mathrm{c}$ & $5.60 \mathrm{~b}$ & $4.50 \mathrm{~d}$ & $5.03 \mathrm{~b}$ & $6.14 \mathrm{c}$ & $7.05 \mathrm{~b}$ & $6.05 \mathrm{c}$ & $6.41 \mathrm{~b}$ \\
\hline 100 ppm & $4.25 \mathrm{de}$ & $4.52 \mathrm{~d}$ & $4.45 \mathrm{~d}$ & $4.41 \mathrm{c}$ & $5.53 \mathrm{~d}$ & $5.58 \mathrm{~d}$ & $5.47 \mathrm{~d}$ & $5.53 \mathrm{c}$ \\
\hline 150 ppm & $4.07 \mathrm{e}$ & $2.93 \mathrm{f}$ & $2.66 \mathrm{f}$ & $3.22 \mathrm{~d}$ & $4.42 \mathrm{e}$ & $3.96 \mathrm{f}$ & $3.20 \mathrm{~g}$ & $3.86 \mathrm{~d}$ \\
\hline \multirow[t]{2}{*}{ Mean (A) } & $4.88 \mathrm{a}$ & $4.84 \mathrm{a}$ & $4.28 \mathrm{~b}$ & & $5.78 \mathrm{~b}$ & $6.03 \mathrm{a}$ & $5.22 \mathrm{c}$ & \\
\hline & \multicolumn{8}{|c|}{ Number of leaves/plant } \\
\hline 0 ppm & $33.00 \mathrm{~b}$ & $37.11 \mathrm{a}$ & $33.18 \mathrm{~b}$ & $34.43 \mathrm{a}$ & $35.33 \mathrm{~b}$ & $39.23 \mathrm{a}$ & $34.16 \mathrm{c}$ & $36.24 \mathrm{a}$ \\
\hline 50 ppm & $27.71 \mathrm{f}$ & $32.83 \mathrm{~b}$ & $31.62 \mathrm{c}$ & $30.72 \mathrm{~b}$ & $28.77 \mathrm{~g}$ & $34.29 \mathrm{c}$ & $33.00 \mathrm{~d}$ & $32.02 \mathrm{~b}$ \\
\hline 100 ppm & $24.54 \mathrm{~g}$ & $29.61 \mathrm{~d}$ & $28.63 \mathrm{e}$ & $27.60 \mathrm{c}$ & $25.70 \mathrm{~h}$ & $32.06 \mathrm{e}$ & $29.71 \mathrm{f}$ & $29.16 \mathrm{c}$ \\
\hline 150 ppm & $24.02 \mathrm{~g}$ & $22.36 \mathrm{~h}$ & $18.70 \mathrm{i}$ & $21.69 \mathrm{~d}$ & $25.13 \mathrm{~h}$ & $23.60 \mathrm{i}$ & $19.24 \mathrm{j}$ & $22.66 \mathrm{~d}$ \\
\hline \multirow[t]{2}{*}{ Mean (A) } & $27.32 \mathrm{c}$ & $30.48 \mathrm{a}$ & $28.03 \mathrm{~b}$ & & $28.73 \mathrm{~b}$ & $32.30 \mathrm{a}$ & $29.03 \mathrm{~b}$ & \\
\hline & \multicolumn{8}{|c|}{ Leaf area $\left(\mathrm{cm}^{2}\right)$} \\
\hline 0 ppm & $6.04 \mathrm{a}$ & $6.08 \mathrm{a}$ & $5.46 \mathrm{~b}$ & $5.86 \mathrm{a}$ & $6.37 \mathrm{a}$ & $6.50 \mathrm{a}$ & $6.11 \mathrm{a}$ & $6.33 \mathrm{a}$ \\
\hline 50 ppm & $5.05 \mathrm{~cd}$ & $5.35 \mathrm{bc}$ & 4.51 ef & $4.97 \mathrm{~b}$ & $6.09 \mathrm{a}$ & $5.17 \mathrm{~b}$ & $4.96 \mathrm{bc}$ & $5.41 \mathrm{~b}$ \\
\hline 100 ppm & $4.80 \mathrm{de}$ & $4.47 \mathrm{f}$ & $4.35 \mathrm{f}$ & $4.54 \mathrm{c}$ & $4.60 \mathrm{~b}-\mathrm{d}$ & $4.79 b-d$ & $4.38 \mathrm{~cd}$ & $4.59 \mathrm{c}$ \\
\hline 150 ppm & 4.54 ef & $3.77 \mathrm{~g}$ & $2.83 \mathrm{~h}$ & $3.71 \mathrm{~d}$ & $4.23 \mathrm{~d}$ & $4.46 \mathrm{~cd}$ & $3.48 \mathrm{e}$ & $4.06 \mathrm{~d}$ \\
\hline Mean (A) & $5.11 \mathrm{a}$ & $4.92 \mathrm{~b}$ & $4.29 \mathrm{c}$ & & $5.32 \mathrm{a}$ & $5.23 \mathrm{a}$ & $4.73 \mathrm{~b}$ & \\
\hline
\end{tabular}

Means followed by the same letters for either pinching treatments, PBZ concentrations or their interaction are not significantly different at the 0.05 probability level according to Duncan's multiple range test. 
Table 3: Effect of pinching treatments, PBZ concentrations and their interactions on some root parameters of Ruellia simplex plants during 2018 and 2019 seasons.

\begin{tabular}{|c|c|c|c|c|c|c|c|c|}
\hline \multirow{3}{*}{$\begin{array}{c}\text { PBZ } \\
\text { concentrations } \\
\text { (B) }\end{array}$} & \multicolumn{4}{|c|}{ Pinching (A) } & \multicolumn{4}{|c|}{ Pinching (A) } \\
\hline & Non & Single & Double & Mean (B) & Non & Single & Double & Mean (B) \\
\hline & \multicolumn{4}{|c|}{2018} & \multicolumn{4}{|c|}{2019} \\
\hline & \multicolumn{8}{|c|}{ Root length (cm) } \\
\hline 0 ppm & $84.00 \mathrm{a}$ & $74.85 \mathrm{~cd}$ & $73.96 \mathrm{c}-\mathrm{e}$ & $77.61 \mathrm{a}$ & $87.44 \mathrm{a}$ & $78.52 \mathrm{~b}-\mathrm{d}$ & $78.44 \mathrm{~b}-\mathrm{d}$ & $81.47 \mathrm{a}$ \\
\hline 50 ppm & $78.37 \mathrm{~b}$ & $73.67 \mathrm{c}-\mathrm{e}$ & $73.04 \mathrm{de}$ & $75.03 \mathrm{~b}$ & $82.81 \mathrm{ab}$ & $76.85 \mathrm{c}-\mathrm{e}$ & $75.70 \mathrm{c}-\mathrm{e}$ & $78.46 \mathrm{~b}$ \\
\hline 100 ppm & $76.74 \mathrm{bc}$ & $71.22 \mathrm{ef}$ & $63.67 \mathrm{~g}$ & $70.54 \mathrm{c}$ & $79.85 \mathrm{bc}$ & $73.74 \mathrm{de}$ & $72.78 \mathrm{e}$ & $75.46 \mathrm{c}$ \\
\hline $150 \mathrm{ppm}$ & $68.18 \mathrm{f}$ & $63.70 \mathrm{~g}$ & $58.93 \mathrm{~h}$ & $63.60 \mathrm{~d}$ & $76.59 \mathrm{c}-\mathrm{e}$ & $66.37 \mathrm{f}$ & $63.82 \mathrm{f}$ & $68.93 \mathrm{~d}$ \\
\hline \multirow[t]{2}{*}{ Mean (A) } & $76.82 \mathrm{a}$ & $70.86 \mathrm{~b}$ & $67.40 \mathrm{c}$ & & $81.68 \mathrm{a}$ & $73.87 \mathrm{~b}$ & $72.68 \mathrm{~b}$ & \\
\hline & \multicolumn{8}{|c|}{ Roots fresh weight (g) } \\
\hline 0 ppm & $13.71 \mathrm{ab}$ & $14.30 \mathrm{a}$ & $12.80 \mathrm{ab}$ & $13.61 \mathrm{a}$ & $14.77 \mathrm{~cd}$ & $17.62 \mathrm{a}$ & $16.58 \mathrm{ab}$ & $16.32 \mathrm{a}$ \\
\hline 50 ppm & $9.98 \mathrm{~cd}$ & $12.81 \mathrm{ab}$ & $11.80 \mathrm{bc}$ & $11.53 \mathrm{~b}$ & $12.03 \mathrm{e}$ & $15.40 \mathrm{bc}$ & $14.79 \mathrm{~cd}$ & $14.07 \mathrm{~b}$ \\
\hline 100 ppm & $9.24 \mathrm{de}$ & $12.46 \mathrm{ab}$ & $11.83 \mathrm{bc}$ & $11.18 \mathrm{~b}$ & 11.79 ef & $13.78 \mathrm{~d}$ & $13.62 \mathrm{~d}$ & $13.06 \mathrm{c}$ \\
\hline 150 ppm & $9.14 \mathrm{de}$ & $7.94 \mathrm{de}$ & $7.45 \mathrm{e}$ & $8.18 \mathrm{c}$ & $11.02 \mathrm{ef}$ & $10.67 \mathrm{ef}$ & $10.35 \mathrm{f}$ & $10.68 \mathrm{~d}$ \\
\hline \multirow[t]{2}{*}{ Mean (A) } & $10.52 \mathrm{~b}$ & $11.88 \mathrm{a}$ & $10.97 \mathrm{ab}$ & & $12.40 \mathrm{~b}$ & $14.37 \mathrm{a}$ & $13.83 \mathrm{a}$ & \\
\hline & \multicolumn{8}{|c|}{ Roots dry weight (g) } \\
\hline 0 ppm & $3.06 \mathrm{e}$ & $4.29 \mathrm{a}$ & $3.54 \mathrm{~cd}$ & $3.63 \mathrm{a}$ & $4.77 \mathrm{~b}$ & $5.83 \mathrm{a}$ & $4.71 \mathrm{~b}$ & $5.10 \mathrm{a}$ \\
\hline 50 ppm & $2.97 \mathrm{e}$ & $3.99 \mathrm{ab}$ & $3.13 \mathrm{de}$ & $3.37 \mathrm{~b}$ & $4.48 \mathrm{~b}$ & $4.68 \mathrm{~b}$ & $4.40 \mathrm{bc}$ & $4.52 \mathrm{~b}$ \\
\hline 100 ppm & $2.82 \mathrm{ef}$ & $3.70 \mathrm{bc}$ & $2.77 \mathrm{ef}$ & $3.10 \mathrm{c}$ & $3.73 \mathrm{~cd}$ & $4.47 \mathrm{~b}$ & $3.56 \mathrm{~d}$ & $3.92 \mathrm{c}$ \\
\hline 150 ppm & $2.70 \mathrm{ef}$ & $2.51 \mathrm{f}$ & $2.07 \mathrm{~g}$ & $2.42 \mathrm{~d}$ & $3.04 \mathrm{de}$ & $3.58 \mathrm{~d}$ & $2.54 \mathrm{e}$ & $3.05 \mathrm{~d}$ \\
\hline Mean (A) & $2.89 \mathrm{~b}$ & $3.62 \mathrm{a}$ & $2.88 \mathrm{~b}$ & & $4.00 \mathrm{~b}$ & $4.64 \mathrm{a}$ & $3.80 \mathrm{~b}$ & \\
\hline
\end{tabular}

Means followed by the same letters for either pinching treatments, PBZ concentrations or their interaction are not significantly different at the 0.05 probability level according to Duncan's multiple range test.

\section{Flowering parameters:}

It was noticed that, flowering parameters were significantly influenced by the different pinching and PBZ spraying treatments as shown in Table (4).

Single pinching significantly reduced the required number of days to flowering (119.80 and 122.50 days in both seasons, respectively) and increased the number of flowers/plant (4.26 and 4.50 flowers in both seasons, respectively). Although, non-pinching treatment extended the period to flowering (132.60 and 136.00 days in both seasons, respectively), this treatment increased flower corolla diameter to the highest values ( 27.38 and $31.63 \mathrm{~mm}$ in both seasons, respectively).

It could be also observed that foliar spraying with PBZ delayed flowering and produced the biggest flowers, in this regard spraying PBZ at $0 \mathrm{ppm}$ (control) accelerated flowering (114.40 and 118.70 days, in both seasons, respectively) and produced the highest flower corolla diameter (31.74 and $31.41 \mathrm{~mm}$ ) in both seasons respectively compared to other PBZ concentrations.

Although the highest number of flowers/plant was obtained by spraying with PBZ at $50 \mathrm{ppm}$ (3.78 and 3.91), in both seasons respectively. The highest number of days required to flowering, the lowest number of flowers/plant and the lowest flower corolla diameter were obtained by spraying with PBZ at $150 \mathrm{ppm}$ in both seasons.

As for the interaction effect of pinching and PBZ concentrations treatments on flowering parameters it could be observed that single pinching + spraying with distilled water only (PBZ at 0 ppm) significantly accelerated flowering as recorded 107.30 and 110.40 days in the first and second seasons, respectively. On the contrary, non-pinching + spraying with PBZ at $150 \mathrm{ppm}$ significantly delayed flowering (143.40 and 145.40 days) in both seasons, respectively. On the other hand, nonpinching + PBZ at 0 ppm produced the highest flower corolla diameter $(33.58$ and $40.97 \mathrm{~mm}$ ), and the single pinching + spraying with $\mathrm{PBZ}$ at $50 \mathrm{ppm}$ produced the highest number of flowers/plant $(5.41$ and 5.67, in both seasons respectively).

It can be observed that, combined treatment of single pinching + spraying with PBZ at 100 ppm delayed flowering by 5 days only than untreated plants as recorded 120.30 days (untreated plants 
recorded 115.90 days) in the first season, while this treatment was approximately equal to untreated plants in the second one.

Table 4: Effect of pinching treatments, PBZ concentrations and their interactions on some flowering parameters of Ruellia simplex plants during 2018 and 2019 seasons.

\begin{tabular}{|c|c|c|c|c|c|c|c|c|}
\hline \multirow{3}{*}{$\begin{array}{c}\text { PBZ } \\
\text { concentrations } \\
\text { (B) }\end{array}$} & \multicolumn{4}{|c|}{ Pinching (A) } & \multicolumn{4}{|c|}{ Pinching (A) } \\
\hline & Non & Single & Double & Mean (B) & Non & Single & Double & Mean (B) \\
\hline & \multicolumn{4}{|c|}{2018} & \multicolumn{4}{|c|}{2019} \\
\hline & \multicolumn{8}{|c|}{ Number of days to flowering (days) } \\
\hline 0 ppm & $115.90 \mathrm{i}$ & $107.30 \mathrm{j}$ & $120.00 \mathrm{gh}$ & $114.40 \mathrm{~d}$ & $123.00 \mathrm{e}$ & $110.40 \mathrm{f}$ & $122.70 \mathrm{e}$ & $118.70 \mathrm{~d}$ \\
\hline 50 ppm & $130.80 \mathrm{e}$ & $118.80 \mathrm{~h}$ & $123.00 \mathrm{f}$ & $124.20 \mathrm{c}$ & $134.40 \mathrm{~cd}$ & $121.80 \mathrm{e}$ & $123.40 \mathrm{e}$ & $126.50 \mathrm{c}$ \\
\hline 100 ppm & $140.50 \mathrm{c}$ & $120.30 \mathrm{~g}$ & $129.90 \mathrm{e}$ & $130.20 \mathrm{~b}$ & $141.30 \mathrm{~b}$ & $122.70 \mathrm{e}$ & $133.00 \mathrm{~d}$ & $132.30 \mathrm{~b}$ \\
\hline 150 ppm & $143.40 \mathrm{a}$ & $132.90 \mathrm{~d}$ & $142.00 \mathrm{~b}$ & $139.40 \mathrm{a}$ & $145.40 \mathrm{a}$ & $135.00 \mathrm{c}$ & $141.60 \mathrm{~b}$ & $140.70 \mathrm{a}$ \\
\hline \multirow[t]{2}{*}{$\operatorname{Mean}(A)$} & $132.60 \mathrm{a}$ & $119.80 \mathrm{c}$ & $128.70 \mathrm{~b}$ & & $136.00 \mathrm{a}$ & $122.50 \mathrm{c}$ & $130.20 \mathrm{~b}$ & \\
\hline & \multicolumn{8}{|c|}{ Number of flowers/plant } \\
\hline 0 ppm & $3.56 \mathrm{c}$ & $4.19 \mathrm{~b}$ & $2.15 \mathrm{ef}$ & $3.30 \mathrm{~b}$ & $3.82 \mathrm{c}$ & $4.52 \mathrm{~b}$ & $2.33 \mathrm{de}$ & $3.56 \mathrm{a}$ \\
\hline 50 ppm & $3.89 \mathrm{bc}$ & $3.74 \mathrm{c}$ & $3.70 \mathrm{c}$ & $3.78 \mathrm{a}$ & $3.96 \mathrm{bc}$ & $3.93 \mathrm{bc}$ & $3.85 \mathrm{c}$ & $3.91 \mathrm{a}$ \\
\hline 100 ppm & $2.37 \mathrm{ef}$ & $5.41 \mathrm{a}$ & $2.78 \mathrm{~d}$ & $3.52 \mathrm{~b}$ & $2.70 \mathrm{~d}$ & $5.67 \mathrm{a}$ & $2.89 \mathrm{~d}$ & $3.75 \mathrm{a}$ \\
\hline 150 ppm & $2.04 \mathrm{f}$ & $3.70 \mathrm{c}$ & $2.44 \mathrm{de}$ & $2.73 \mathrm{c}$ & $2.07 \mathrm{e}$ & $3.89 \mathrm{c}$ & $2.52 \mathrm{de}$ & $2.83 \mathrm{~b}$ \\
\hline \multirow[t]{2}{*}{ Mean (A) } & $2.96 \mathrm{~b}$ & $4.26 \mathrm{a}$ & $2.77 \mathrm{~b}$ & & $3.14 \mathrm{~b}$ & $4.50 \mathrm{a}$ & $2.90 \mathrm{~b}$ & \\
\hline & \multicolumn{8}{|c|}{ Flower corolla diameter $(\mathrm{mm})$} \\
\hline 0 ppm & $33.58 \mathrm{a}$ & $32.27 \mathrm{a}$ & $29.38 \mathrm{~b}$ & $31.74 \mathrm{a}$ & $40.97 \mathrm{a}$ & $35.72 \mathrm{~b}$ & $35.55 \mathrm{~b}$ & $37.41 \mathrm{a}$ \\
\hline 50 ppm & $27.28 \mathrm{bc}$ & $25.97 \mathrm{~cd}$ & $24.63 \mathrm{de}$ & $25.96 \mathrm{~b}$ & $33.70 \mathrm{bc}$ & $33.28 \mathrm{bc}$ & $31.37 \mathrm{c}$ & $32.78 \mathrm{~b}$ \\
\hline 100 ppm & $25.67 \mathrm{~cd}$ & $25.25 \mathrm{c}-\mathrm{e}$ & $22.90 \mathrm{ef}$ & $24.61 \mathrm{~b}$ & $27.83 \mathrm{~d}$ & $26.15 \mathrm{de}$ & $26.40 \mathrm{de}$ & $26.79 \mathrm{c}$ \\
\hline 150 ppm & $23.00 \mathrm{ef}$ & $21.52 \mathrm{f}$ & $18.65 \mathrm{~g}$ & $21.06 \mathrm{c}$ & $24.23 \mathrm{e}$ & $21.22 \mathrm{f}$ & $19.55 \mathrm{f}$ & $21.67 \mathrm{~d}$ \\
\hline Mean (A) & $27.38 \mathrm{a}$ & $26.25 \mathrm{a}$ & $23.89 \mathrm{~b}$ & & $31.68 \mathrm{a}$ & $29.09 \mathrm{~b}$ & $28.22 \mathrm{~b}$ & \\
\hline
\end{tabular}

Means followed by the same letters for either pinching treatments, PBZ concentrations or their interaction are not significantly different at the 0.05 probability level according to Duncan's multiple range test.

\section{Pigments content:}

As presented in Table (5), double pinching significantly produced the highest chlorophyll "a" content $(0.812 \mathrm{mg} / \mathrm{g}$ f.w. $)$, while single pinching produced the highest chlorophylls " $\mathrm{b}$ " and carotenoids contents $(0.236$ and $0.391 \mathrm{mg} / \mathrm{g}$ f.w., respectively). The lowest values in terms of chlorophyll "a" $(0.755 \mathrm{mg} / \mathrm{g}$ f.w. $)$ and carotenoids contents $(0.319 \mathrm{mg} / \mathrm{g}$ f.w. $)$ were obtained by nonpinching treatment.

Regarding spraying with PBZ, there was a significant effect on pigments content. Spraying with PBZ at 100 ppm produced the highest chlorophyll "a", "b" and carotenoids contents $(0.876,0.287$ and $0.393 \mathrm{mg} / \mathrm{g}$ f.w., respectively). In contrast, spraying with the highest PBZ concentration produced the lowest chlorophyll "a" and "b" (0.676 and $0.152 \mathrm{mg} / \mathrm{g}$ f.w., respectively). On the other hand, the lowest carotenoids content was obtained by spraying with distilled water only.

As for the effect of interaction treatments, a significant effect was observed on pigments content of $R$. simplex plants as a result to applying different treatments. The highest significant values of chlorophyll "a", "b" and carotenoids (0.939, 0.329 and $0.415 \mathrm{mg} / \mathrm{g}$ f.w., respectively) were obtained by single pinching + spraying with PBZ at $100 \mathrm{ppm}$. In contrast, the lowest values of chlorophyll "a" $(0.568 \mathrm{mg} / \mathrm{g}$ f.w. $)$, chlorophyll "b" (0.144 mg/g f.w.) and carotenoids content $(0.268 \mathrm{mg} / \mathrm{g}$ f.w. $)$ were obtained by single pinching + PBZ at $150 \mathrm{ppm}$, double pinching + PBZ at $150 \mathrm{ppm}$ and non-pinching + spraying with PBZ at $0 \mathrm{ppm}$ (control), respectively. 
Table 5: Effect of pinching treatments, PBZ concentrations and their interactions on pigments content of Ruellia simplex leaves at the end of 2019 season.

\begin{tabular}{|c|c|c|c|c|}
\hline \multirow{3}{*}{$\begin{array}{c}\text { PBZ } \\
\text { concentrations } \\
\text { (B) }\end{array}$} & \multicolumn{4}{|c|}{ Pinching (A) } \\
\hline & Non & Single & Double & Mean (B) \\
\hline & \multicolumn{4}{|c|}{ Chlorophyll "a" (mg/g f.w.) } \\
\hline 0 ppm & $0.752 \mathrm{~h}$ & $0.738 \mathrm{i}$ & $0.797 \mathrm{f}$ & $0.762 \mathrm{c}$ \\
\hline 50 ppm & $0.872 \mathrm{~b}$ & $0.857 \mathrm{c}$ & $0.813 \mathrm{e}$ & $0.847 \mathrm{~b}$ \\
\hline 100 ppm & $0.828 \mathrm{~d}$ & $0.939 \mathrm{a}$ & $0.860 \mathrm{c}$ & $0.876 \mathrm{a}$ \\
\hline 150 ppm & $0.568 \mathrm{k}$ & $0.681 \mathrm{j}$ & $0.779 \mathrm{~g}$ & $0.676 \mathrm{~d}$ \\
\hline \multirow[t]{2}{*}{ Mean (A) } & $0.755 \mathrm{c}$ & $0.804 \mathrm{~b}$ & $0.812 \mathrm{a}$ & \\
\hline & \multicolumn{4}{|c|}{ Chlorophyll "b" (mg/g f.w.) } \\
\hline 0 ppm & $0.172 \mathrm{c}$ & $0.191 \mathrm{c}$ & $0.152 \mathrm{c}$ & $0.171 \mathrm{c}$ \\
\hline 50 ppm & $0.297 \mathrm{ab}$ & $0.274 \mathrm{~b}$ & $0.188 \mathrm{c}$ & $0.253 \mathrm{~b}$ \\
\hline 100 ppm & $0.270 \mathrm{~b}$ & $0.329 \mathrm{a}$ & $0.262 \mathrm{~b}$ & $0.287 \mathrm{a}$ \\
\hline 150 ppm & $0.162 \mathrm{c}$ & $0.150 \mathrm{c}$ & $0.144 \mathrm{c}$ & $0.152 \mathrm{c}$ \\
\hline \multirow[t]{2}{*}{ Mean (A) } & $0.225 \mathrm{a}$ & $0.236 \mathrm{a}$ & $0.186 \mathrm{~b}$ & \\
\hline & \multicolumn{4}{|c|}{ Carotenoids content (mg/g f.w.) } \\
\hline 0 ppm & $0.268 \mathrm{f}$ & $0.343 \mathrm{~cd}$ & $0.342 \mathrm{c}-\mathrm{e}$ & $0.318 \mathrm{c}$ \\
\hline 50 ppm & $0.329 \mathrm{de}$ & $0.399 \mathrm{a}$ & $0.352 \mathrm{bc}$ & $0.360 \mathrm{~b}$ \\
\hline 100 ppm & $0.351 \mathrm{bc}$ & $0.415 \mathrm{a}$ & $0.413 \mathrm{a}$ & $0.393 \mathrm{a}$ \\
\hline 150 ppm & $0.326 \mathrm{e}$ & $0.405 \mathrm{a}$ & $0.368 \mathrm{~b}$ & $0.366 \mathrm{~b}$ \\
\hline Mean (A) & $0.319 \mathrm{c}$ & $0.391 \mathrm{a}$ & $0.369 \mathrm{~b}$ & \\
\hline
\end{tabular}

Means followed by the same letters for either pinching treatments, PBZ concentrations or their interaction are not significantly different at the 0.05 probability level according to Duncan's multiple range test.

\section{Chemical composition:}

As shown in Table (6), it could be noticed that single pinching seems to be more effective than non or double pinching in increasing total carbohydrates in shoots and roots and in total indoles to the highest significant values as recorded $21.26 \%, 23.82 \%$ and $3.04 \mathrm{mg} / 100 \mathrm{~g}$ d.w., respectively. The highest significant total phenols content was obtained by double pinching $(0.147 \%)$, this was accompanied with the highest reduction of total carbohydrates in shoots $(17.08 \%)$ and roots $(20.80 \%)$.

As for the effect of PBZ concentrations, PBZ at 0 ppm (control) increased total carbohydrates in both shoots and roots to the highest significant values (22.69 and $26.13 \%$, respectively), this treatment reduced total indoles and phenols to the lowest values $(1.81 \mathrm{mg} / 100 \mathrm{~g}$ d.w. and $0.105 \%$, respectively). The highest concentration of PBZ ( $150 \mathrm{ppm})$ reduced total carbohydrates in either shoots and roots to the lowest significant values (15.37 and $18.82 \%$, respectively), this was accompanied with producing the highest significant phenols content as recorded $0.161 \%$. On the other hand, the highest total indoles content was achieved with spraying with PBZ at $100 \mathrm{ppm}$ as recorded $3.53 \mathrm{mg} / 100 \mathrm{~g}$ d.w.

Regarding the effect of combined treatments on chemical composition it could be observed that single pinching + spraying with distilled water only (PBZ at $0 \mathrm{ppm}$ ) significantly increased total carbohydrates in shoots and roots to the highest significant values (27.39 and $31.59 \%$, respectively). In contrast, the lowest values were obtained by double pinching + spraying with the highest PBZ concentration (150 ppm) as recorded 14.91 and $17.22 \%$, respectively. In this concern, single pinching + spraying with PBZ at $100 \mathrm{ppm}$ resulted in the highest total indoles content $(4.38 \mathrm{mg} / 100 \mathrm{~g}$ d.w.) when compared with non-pinching + PBZ at $0 \mathrm{ppm}$ which gave the lowest values $(1.14 \mathrm{mg} / 100 \mathrm{~g}$ d.w.). On the other hand, the highest values of total phenols were obtained by double pinching + spraying with the highest PBZ concentration (150 ppm) as recorded $0.224 \%$.

It is clear from the obtained results that pinching showed an effective role in shortening height of the treated plants with producing more branches and flowers in addition to more pigment's formation, single pinching demonstrated the superiority in this concern. Such effect was enhanced by combining pinching with PBZ at 50 or 100 ppm, thereby an optimum dwarfism was obtained. These desired effects were accompanied with delaying flowering, the only exception was obtained by single 
pinching without spraying with PBZ which significantly accelerated flowering. Not only morphological traits were influenced by both pinching and PBZ but also chemical constituents i.e. pigment contents, total carbohydrates, indoles and phenols. In this regard, chlorophylls ( $a$ and $b$ ), carotenoids, indoles and phenols were enhanced by pinching and PBZ, while, the mastery was to single pinching only in terms of total carbohydrates in both shoots and leaves. Furthermore, this study emphasized the applied role of PBZ as a plant growth retardant in controlling growth of plants especially for floriculture industry.

Table 6: Effect of pinching treatments, PBZ concentrations and their interactions on some chemical constituents of Ruellia simplex plants at the end of 2019 season.

\begin{tabular}{|c|c|c|c|c|c|c|c|c|}
\hline \multirow{3}{*}{$\begin{array}{c}\text { PBZ } \\
\text { concentrations } \\
\text { (B) }\end{array}$} & \multicolumn{4}{|c|}{ Pinching (A) } & \multicolumn{4}{|c|}{ Pinching (A) } \\
\hline & Non & Single & Double & Mean (B) & Non & Single & Double & Mean (B) \\
\hline & \multicolumn{4}{|c|}{ Total carbohydrates of shoots (\%) } & \multicolumn{4}{|c|}{ Total carbohydrates of roots (\%) } \\
\hline 0 ppm & $21.56 \mathrm{c}$ & $27.39 \mathrm{a}$ & $19.13 \mathrm{de}$ & $22.69 \mathrm{a}$ & $23.54 \mathrm{~b}$ & $31.59 \mathrm{a}$ & $23.25 \mathrm{bc}$ & $26.13 \mathrm{a}$ \\
\hline 50 ppm & $21.73 \mathrm{c}$ & $22.70 \mathrm{~b}$ & $18.49 \mathrm{e}$ & $20.97 \mathrm{~b}$ & $22.69 \mathrm{bc}$ & $23.27 \mathrm{bc}$ & $22.98 \mathrm{bc}$ & $22.98 \mathrm{~b}$ \\
\hline 100 ppm & $17.53 \mathrm{f}$ & $19.57 \mathrm{~d}$ & $15.78 \mathrm{~g}$ & $17.63 \mathrm{c}$ & 20.60 ef & $22.13 \mathrm{~cd}$ & $19.74 \mathrm{f}$ & $20.82 \mathrm{c}$ \\
\hline 150 ppm & $15.81 \mathrm{~g}$ & $15.38 \mathrm{gh}$ & $14.91 \mathrm{~h}$ & $15.37 \mathrm{~d}$ & 20.98 de & $18.27 \mathrm{~g}$ & $17.22 \mathrm{~g}$ & $18.82 \mathrm{~d}$ \\
\hline \multirow[t]{2}{*}{ Mean (A) } & $19.16 \mathrm{~b}$ & $21.26 \mathrm{a}$ & $17.08 \mathrm{c}$ & & $21.95 \mathrm{~b}$ & $23.82 \mathrm{a}$ & $20.80 \mathrm{c}$ & \\
\hline & \multicolumn{4}{|c|}{ Total indoles (mg/100 g d.w.) } & \multicolumn{4}{|c|}{ Total phenols (\%) } \\
\hline 0 ppm & $1.14 \mathrm{f}$ & $2.47 \mathrm{~cd}$ & $1.81 \mathrm{e}$ & $1.81 \mathrm{c}$ & $0.091 \mathrm{e}$ & $0.108 \mathrm{de}$ & $0.116 \mathrm{~cd}$ & $0.105 \mathrm{c}$ \\
\hline 50 ppm & $2.28 \mathrm{~d}$ & $2.84 \mathrm{bc}$ & $2.23 \mathrm{~d}$ & $2.45 \mathrm{~b}$ & $0.097 \mathrm{e}$ & $0.115 \mathrm{~cd}$ & $0.123 \mathrm{~b}-\mathrm{d}$ & $0.112 \mathrm{c}$ \\
\hline 100 ppm & $3.09 \mathrm{~b}$ & $4.38 \mathrm{a}$ & $3.12 \mathrm{~b}$ & $3.53 \mathrm{a}$ & $0.119 \mathrm{~b}-\mathrm{d}$ & $0.122 \mathrm{~b}-\mathrm{d}$ & $0.125 \mathrm{bc}$ & $0.122 \mathrm{~b}$ \\
\hline 150 ppm & $1.82 \mathrm{e}$ & $2.48 \mathrm{~cd}$ & $1.50 \mathrm{ef}$ & $1.93 \mathrm{c}$ & $0.123 \mathrm{~b}-\mathrm{d}$ & $0.136 \mathrm{~b}$ & $0.224 \mathrm{a}$ & $0.161 \mathrm{a}$ \\
\hline Mean (A) & $2.08 \mathrm{~b}$ & $3.04 \mathrm{a}$ & $2.17 \mathrm{~b}$ & & $0.108 \mathrm{c}$ & $0.120 \mathrm{~b}$ & $0.147 \mathrm{a}$ & \\
\hline
\end{tabular}

Means followed by the same letters for either pinching treatments, PBZ concentrations or their interaction are not significantly different at the 0.05 probability level according to Duncan's multiple range test.

Referring to the effect of pinching treatments, the obtained results were close to those obtained by Gibson et al. (2003) on Argyranthemum frutescens, Saiyad et al. (2010) on gaillardia, Shanan and Soliman (2011) on snapdragon, Rezazadeh and Harkess (2015) on Odontonema callistachyum and Vasoya et al. (2015) on gaillardia. In this aspect, Rathore and Mishra (2014) on Tagetes erecta L. cv. Pusa Basanti Gainda revealed that pinching significantly decreased the plant height and increased number of primary branches with more flower's number/plant. Abou-Dahab et al. (2015) on Russelia equisetiformis, found that both one and two pinching treatments reduced plant height and total indoles, but one pinching treatment increased number of shoots and flowers/plant, roots fresh and dry weights, chlorophylls "a", "b", carotenoids and total phenols, while, two pinching treatment reduced fresh and dry weights of shoots and roots and total indoles. El-Sadek (2016) reported that pinching Hibiscus rosa-sinensis, L. cv. Yellow reduced plant height, fresh and dry weights of vegetative parts and roots and increased chlorophylls "a", "b", carotenoids and indoles. Finally, Abd El-Aal and Mohamed (2017) concluded that applying pinching on Pelargonium zonale plants reduced plant height, leaf area and chlorophylls content and delayed flowers, while number of branches, leaves and flowers/plant were increased.

Positive effects of pinching on growth could be explained by that keeping apical meristem and young expanding leaves which containing a metabolic sink and auxin source that inhibit the outgrowth of lateral buds (Weiss and Shilo, 1988), removal of shoot apex by pinching the growing tip, removes the source of apical dominance and assimilates are diverted into lateral buds, for this reason upward growth is depressed and branching is occurred resulting in compact plants (Cline, 1991). Therefore, as leaf area of pinched plants is reduced, pigments in leaf tissues are concentrated, so its contents increased. The leaves of both treated and untreated plants possibly contain the same number of cells, but because the cells in leaves of treated plants are smaller, the chlorophyll is more concentrated in the reduced cell volume (Nazarudin, 2012).

Regarding the effect of PBZ the obtained results revealed that foliar spraying with PBZ at 50 or 100 ppm was more effective than PBZ at 150 ppm on most studied traits particularly for increasing 
number of branches and flowers/plant, chlorophylls "a", "b" and carotenoids and indoles. Although high PBZ concentration $(150 \mathrm{ppm})$ in the present study produced the highest reduction in plant height, this was accompanied with undesirable traits e.g. reduced fresh and dry weights of both vegetative parts and roots, reduced the number of leaves and flowers/plant and delayed flowering to the longest period. This suggested that solutions containing PBZ at $150 \mathrm{ppm}$ are too concentrated to achieve the desired results.

These results were in harmony with those obtained by Maus (1987) on Hibiscus rosa-sinensis, Villegas and Lozoya (1992) on poinsettia, Wilfret and Barrett (1995) on Rhododendron obtusum, Gad et al. (1997) on Fuchsia magellanica cv. Beacon, El-Sallami (2001) on potted poinsettia, Warner and Erwin (2003) on Hibiscus coccineus, H. radiatus and H. trionum, Matsoukis et al. (2004) on Lantana camara, Meijón et al. (2009) on Azalea japonica and Saiyad et al. (2010) and Vasoya et al. (2015) on gaillardia, Shanan and Soliman (2011) on snapdragon, Rathore and Mishra (2014) on Tagetes erecta L., Shahin et al. (2014) on Chrysanthemum carinatum, Mutlu and Agan (2015) on ornamental pepper, El-Sadek (2016) on Hibiscus rosa-sinensis and Mohamed (2016) on Lagerstroemia indica. These results also were close to the results obtained by El-Bably (2008) who revealed that high concentrations of PBZ reduced plant height, leaves number, fresh and dry weights of vegetative parts, root length and fresh and dry weights of roots, total carbohydrates, delayed flowering time, increased phenol and indole contents of Anisacanthus wrightii. Noor El-Deen et al. (2014) reported that PBZ foliar spraying at 50 or $100 \mathrm{ppm}$ reduced plant height, top growth and roots fresh and dry weights and delayed flowering and increased both chlorophyll a, b, carotenoids, total indoles and phenols of Gaillardia pulchella. Abou-Dahab et al. (2015) on Russelia equisetiformis demonstrated that PBZ reduced plant height and root length, but increased number of shoots and flowers/plant, chlorophyll "a", "b", carotenoids and total phenols. Abd El-Aal and Mohamed (2017) recorded that foliar spraying with PBZ caused a decrement in plant height and leaf area of Pelargonium zonale plants, this was accompanied with delaying of flowering and increasing number of branches/plant. Heikal (2017) on Sanchezia nobilis reported that foliar spraying with PBZ reduced plant height, root length, leaf area, number of leaves/plant, shoots and roots fresh and dry weights. Jagdale et al. (2017) reported that increasing PBZ concentration led to reduce plant height and leaf area but increased number of flowers/plant and delayed flowering of annual chrysanthemum.

PBZ reduces stem elongation by inhibiting the kaurene oxidation sequence of reactions in the gibberellin biosynthesis (Warner and Erwin, 2003). The shorter stems of plants treated by PBZ are most likely due to the systematic inhibition of biosynthesis of gibberellins, which are plant growth regulators effective in shoot elongation. PBZ is a potent inhibitor of biosynthesis of gibberellins (Teto et al., 2016). In this regard the application of GA biosynthesis inhibitors caused alterations in levels of polyamines, gibberellin and cytokinins and in global DNA methylation levels during floral transition; also, these changes in plant growth regulators and DNA methylation were correlated with flower development (Meijón et al., 2011). Soumya et al. (2017) revealed that PBZ maintaining relative water content, membrane stability index, photosynthetic activity, photosynthetic pigments and protects the photosynthetic machinery by enhancing the level of osmolytes, antioxidant activities and level of endogenous hormones and thereby enhances optimum growth.

Combined treatments between pinching and PBZ showed a great influence on obtaining optimum stunting of $R$. simplex plants, the obvious combined treatment was single pinching + foliar spraying with PBZ at $100 \mathrm{ppm}$. These results were in parallel to those obtained by Maloupa et al. (2000) on Vitex agnus-castus, Abou-Dahab and Habib (2005) on Barleria cristata plant, Shanan and Soliman (2011) on snapdragon, Abou-Dahab et al. (2015) on Russelia equisetiformis, Mutlu and Agan (2015) on ornamental paper, El-Sadek (2016) on Hibiscus rosa-sinensis and Abd El-Aal and Mohamed (2017) on Pelargonium zonale.

Finally, it is recommended to treat $R$. simplex plants cultivated in 16-cm-pots filled with peat moss + perlite (1:1 by volume) with single pinch after 1 month from transplanting +4 applications at one-month interval with PBZ at $100 \mathrm{ppm}$ for optimum stunting with satisfied quality and aesthetic value as a pot plant.

\section{References}

A.O.A.C., 1980. Official Methods of Analysis of the Association of Official Agricultural Chemists. $15^{\text {th }}$ Ed., Arlington, Virginia, 22201: 877-878. 
Abd El-Aal, M.A. and Y.F.Y. Mohamed, 2017. Effect of pinching and paclobutrazol on growth, flowering, anatomy and chemical compositions of potted geranium (Pelargonium zonal L.) plant. International Journal of Plant \& Soil Science, 17(6): 1-22.

Abou-Dahab, M.A., E.A. Khella and K.A. Emam, 2015. Effect of pinching and paclobutrazol (Pbz) on vegetative growth of Russelia equisetiformis for using as a pot plant. Egyptian Journal of Horticulture, 42(2): 913-930.

Abou-Dahab, T.A.M. and A.M.A. Habib, 2005. Production of Barleria cristata L. as a dwarf flowering pot plant. Annals of Agricultural Sci., Moshtohor, 43(2): 746-727.

Bailey, D., 1998. Height Control of Commercial Greenhouse Flowers. North Carolina State University College of Agriculture \& Life Sciences, USA, Horticulture Information Leaflet 528, $17 \mathrm{p}$.

Bañón, S., J. Miralles, A. Navarro, and M.J. Sánchez-Blanco, 2009. Influence of paclobutrazol and substrate on daily evapotranspiration of potted geranium. Scientia Horticulturae, 122(4): 572578.

Cline, M.G., 1991. Apical dominance. Bot. Rev., 57: 318-358.

Duncan, D.B. 1955. Multiple range and multiple F test. Journal of Biometrics, 11:1-42.

El-Bably, S.Z., 2008. Growth and flowering of Anisacanthus wrightii plant as affected by cycocel and paclobutrazol application. Alexandria Journal of Agricultural Research, 53(1): 73-80.

El-Sadek, Z.H., 2016. Effect of pinching and paclobutrazol on Hibiscus rosa-sinensis, L. cv. "Yellow" plant. Scientific Journal of Flowers and Ornamental Plants, 3(4): 233-244.

El-Sallami, I.H., 2001. Controlling growth habit of potted poinsettia under two light intensity conditions. Assiut Journal of Agricultural Sciences, 32(4): 109-139.

Ferreira, T. and W.S. Rasband, 2012. ImageJ User Guide-IJ-1.46. http://imagej.nih.gov/ij/docs/guide.

Freyre, R. and S.B. Wilson, 2014. Ruellia simplex R10-105-Q54 ('Mayan Pink'). HortScience, 49(4): 499-502.

Gad, M., G. Schmidit and I. Gerzson, 1997. Comparison of application methods of growth retardants on the growth and flowering of Fuchsia magellanica Lam. Horticultural Science, 29(1-2): 7077.

Gibson, J.L., T.J. Cavins, L. Greer, B.E. Whipker and J.M. Dole, 2003. Efficacy of plant growth regulators on the growth of Argyranthemum frutescens 'Comet Pink'. Acta Horticulturae, 624: 213-216.

Gomez, K.A., and A.A. Gomez, 1984. Statistical procedures for agricultural research. John Wiley \& Sons, New York, USA, 680 p.

Heikal, A., 2017. Controlling growth of Sanchezia plant by foliar application of micronutrients and paclobutrazol. Egyptian Journal of Horticulture, 44(2): 127-140.

Heins, RD., B. Liu and E.S. Runkle, 2000. Regulation of crop growth and development based on environmental factors. Acta Horticulturae, 513: 17-26.

Herbert, D., P.J. Phipps and R.E. Strange, 1971. Chemical Analysis of Microbial Cells. In Methods in Microbiology, Academic Press, 5: 209-344.

Jagdale, A.R., Y.R. Khobragade, D.M. Panchbhai, G.N. Ghormade and A.C. Bhaskarwar, 2017. Growth and flowering of annual chrysanthemum influenced by cycocel and paclobutrazol. Journal of Soils and Crops, 27(1): 143-146.

Khandelwal, S.K., N.K. Jain and P. Singh, 2003. Effect of growth retardants and pinching on growth and yield of African marigold. Journal of Ornamental Horticulture, 6(3): 271-273.

Larsen, P., A. Harbo, S. Klungsour and T. Asheim, 1962. On the biogenesis of some indole compounds in Acetobacter xylinum. Physiologia Plantarum, 15(3): 552-565.

Maloupa, E., D. Gerasopoulos, A. Marnasidis, D. Zervaki, 2000. Paclobutrazol and pinching affect visual quality characteristics of potted Vitex agnus-castus plants. Acta Horticulturae, 541: 295298.

Matsoukis, A.S., I. Tsiros and A. Kamoutsis, 2004. Leaf area response of Lantana camara L. subsp. camara to plant growth regulators under different photosynthetic flux conditions. HortScience, 39(5): 1042-1044.

Maus, W.L., 1987. Effect of paclobutrazol and uniconizole-p on Hibiscus rosa-sinensis. Proc. Fla. State Hort. Soc., 100: 373-375. 
Meijón, M., C.M. Jesús, L. Valledor, R. Rodríguez and I. Feito, 2011. Epigenetic and physiological effects of gibberellin inhibitors and chemical pruners on the floral transition of azalea. Physiologia Plantarum, 141(3): 276-288.

Meijón, M., R. Rodríguez, M.J. Cañal and I. Feito, 2009. Improvement of compactness and floral quality in azalea by means of application of plant growth regulators. Scientia Horticulturae, 119(2): 169-176.

Mohamed, N.T., 2016. Effect of Paclobutrazol Concentration, Application Techniques and Frequency on Growth and Flowering of Lagerstroemia indica. M.Sc. Thesis, Fac Agric., Putra Univ., Malaysia, $65 \mathrm{p}$.

MSTAT Development Team, 1989. MSTAT User's Guide: A Microcomputer Program for the Design Management and Analysis of Agronomic Research Experiments. Michigan State University, East Lansing, USA.

Mutlu, S.S. and E. Agan, 2015. Effects of paclobutrazol and pinching on ornamental pepper. HortTechnology, 25(5): 657-664.

Nau, J., 2011. Ball Redbook, Volume 2: Crop Production, $18^{\text {th }}$ Ed. Ball Publishing, West Chicago, Illinois, USA, 785 p.

Nazarudin, A.M.R., 2012. Plant growth retardants effect on growth and flowering of potted Hibiscus rosa-sinensis L. J. Trop. Plant Physiol., 4:29-40.

Noor El-Deen, T.M., B. El-Sayed and S. Shahin, 2014. Chrysanthemum and gaillardia as stunted winter annuals II. Blanketflower (Gaillardia pulchella Foug.). Scientific Journal of Flowers and Ornamental Plants, 1(2): 155-162.

Nurhazwani, M., A. Yahya and I.R. Shairul, 2018. Growth Performance of Ruellia brittoniana as Affected by Daminozide Concentrations. Proc. of $27^{\text {th }}$ Malaysian Society of Plant Physiology Conference, Johor, Malaysia, 25: 93-97.

Rathore, I. and A. Mishra, 2014. Effect of pinching and plant bioregulators on flowering, yield and root characteristics of marigold (Tagetes erecta L.) cv. Pusa Basanti Gainda under sub humid region of Rajasthan. International Journal of Basic and Applied Agricultural Research, 12(3): 369-373.

Rezazadeh, A. and R.L. Harkess, 2015. Effects of pinching, number of cuttings per pot, and plant growth regulators on height control of purple firespike. HortTechnology, 25(1): 71-75.

Saiyad, M.Y., R.G. Jadav, A.B. Parmar and K.M. Chauhan, 2010. Effect of plant growth retardants and pinching on growth, flowering and yield of gaillardia (Gaillardia pulchella Foug.) cv. Lorenziana. The Asian Journal of Horticulture, 5(1): 121-122.

Sehrawat, S.K., D.S. Dahiya, S. Singh and G.S. Rana, 2003. Effect of nitrogen and pinching on the growth, flowering and yield of marigold (Tagetes erecta L.) cv. African Giant Double Orange. Haryana Journal of Horticultural Science, 32(1/2): 59-61.

Shahin, S., T.M. Noor El-Deen and B. El-Sayed, 2014. Chrysanthemum and Gaillardia as stunted winter annuals I. Tricolor chrysanthemum (Chrysanthemum carinatum Schousb.). Scientific Journal of Flowers and Ornamental Plants, 1(2): 145-154.

Shanan, N.T. and A.S. Soliman, 2011. Response of snapdragon plants to pinching and growth retardants treatments. American-Eurasian Journal of Sustainable Agriculture, 5(2): 150-158.

Smith, A.M., S.B. Wilson, C.R. Adams, R. Freyre, K.V.S. Hupp, A.M. Fox and R.K. Stocker, 2010. Natural Area Weeds: Mexican Petunia (Ruellia simplex). U.S. Department of Agriculture, UF/IFAS Extension Service, University of Florida, USA, 7 p.

Soumya, P.R., P. Kumar and M. Pal, 2017. Paclobutrazol: a novel plant growth regulator and multistress ameliorant. Indian Journal of Plant Physiology, 22(3): 267-278.

Teto, A.A., C.P. Laubscher, P.A. Ndakidemi and I. Matimati, 2016. Paclobutrazol retards vegetative growth in hydroponically-cultured Leonotis leonurus (L.) R.Br. Lamiaceae for a multipurpose flowering potted plant. South African Journal of Botany, 106:67-7.

Vasoya, N., V. Ramesh, P. Nitesh and K. Dilip, 2015. Response of gaillardia to plant growth regulators and pinching. Trends in Biosci., 8(16): 4090-4095.

Villegas, T.O. and S.H. Lozoya, 1992. Effect of paclobutrazol (PBZ) on poinsettia (Euphorbia pulcherrima W.) cultivar Gutbier V-10 under greenhouse conditions in Chapingo, Mex. Revista Chapingo, 15(73-74): 77-80 . 
Warner, R.M. and J.E. Erwin, 2003. Effect of plant growth retardants on stem elongation of Hibiscus species. HortTechnology, 13(2): 293-296.

Weiss, D. and R. Shilo, 1988. Axillary bud inhibition induced by young leaves or bract in Euphorbia pulcherrima Willd. Ann. Bot., 62: 435-440.

Wellburn, A.R. and H. Lichtenthaler, 1984. Formulae and program to determine total carotenoids and chlorophylls-a and b of leaf extracts in different solvents. Adv. Agricul. Biotechn., 2(1):9-12.

Wiese, C., C.R. Adams and A.M. Smith, 2013. Experimental research informs control of Mexican petunia (Ruellia simplex) in natural areas and home-gardens. Proceedings of the Florida State Horticultural Society, 126: 332-337.

Wilfret, G.J. and J.E. Barrett, 1995. Effect of growth regulators on growth and flower development of potted azaleas. Proceedings of the Florida State Horticultural Society, 107: 175-177. 